\title{
An Assessment of Polarized Light Microscopy for the Quantification of Grain Size and Orientation in Titanium Alloys via Microanalytical Correlative Light to Electron Microscopy (CLEM)
}

\author{
Hamed Safaie ${ }^{1}$, Ria L. Mitchell ${ }^{1}$, Richard Johnston ${ }^{1}$, James Russell ${ }^{1}$ and Cameron Pleydell-Pearce ${ }^{1}$ \\ 1. Advanced Imaging of Materials (AIM) Facility, College of Engineering, Swansea University, \\ Swansea, UK.
}

The influence of bulk crystallographic texture on the mechanical performance of titanium based alloys is well documented [1]. In parallel, studies on the microtexture of titanium using crystal orientation maps $(\mathrm{COM})$ via electron backscatter diffraction (EBSD) have revealed that bulk texture in these materials is dictated by large areas of common orientation often referred to as either microtexture or macrozones [2]. Consequently, the characterization of texture heterogeneities via EBSD in titanium alloys has received increased attention. However, this technique is both costly and time consuming, limiting its suitability as an industrially viable method of characterizing these effects (in quality assurance for example). Polarized light microscopy (PLM) is ideally suited to the vast majority of commercially available titanium alloys on account of the optically birefringence of the dominant low temperature HCP $\alpha$ phase, providing contrast between units of different crystal orientation. The resulting images can be captured through standard digital imaging equipment providing a qualitative alternative to producing EBSD orientation maps at vastly increased speed and reduced cost. Previous investigations in the biosciences have demonstrated success in quantifying optical birefringence behavior in transmitted light microscopy [3] and other studies have refined the principle through the implementation of laser optics to produce orientation maps [4]. However, a thorough comparison of EBSD and reflected light PLM in titanium alloys has not previously received attention.

Analytical CLEM was employed on four different microstructural variants of the $\alpha / \beta$ titanium alloy Ti-6Al-4V. Light microscopy was performed on a Carl Zeiss Axio Observer in bright field and polarized condition, EBSD data was recorded on a Carl Zeiss Crossbeam 540 with Oxford Instruments EBSD and CLEM was facilitated via the Carl Zeiss Shuttle and Find system. Comparisons were made by imaging / analyzing identical areas via EBSD, PLM (both in the un-etched condition) and bright field optical microscopy (in the etched condition). An example of an initial comparison can be seen in figure 1. This highlighted the inability of etching to distinguish between low and high angle grain boundaries, allowing microtexture inhomogeneity to be 'hidden'. PLM was able to elucidate these features well. Subsequent, quantitative analysis of the plane of polarization (major axis) noted a $180^{\circ}$ symmetry in extinction position (figure 2). This symmetry could be loosely correlated to crystal orientation in these limited examples. To expand the dataset and develop stronger correlations custom correlative image analysis software was developed that permitted a spatially resolved comparison of both techniques across many grains. Early comparisons of COM output data from this software (figure 3) suggests that PLM could present a viable route for quantitative analysis of titanium alloys in future.

References:

[1] M.R. Bache, International Journal of Fatigue 25 (2003), p. 1079.

[2] L. Germain et al, Materials Science and Engineering A319-321 (2001), p. 409.

[3] S. Ross et al, Journal of microscopy 178 Pt.1 (1997), p. 62.

[4] F.Massoumian et al, Journal of Microscopy 209 Pt.1 (2003), p. 13. 


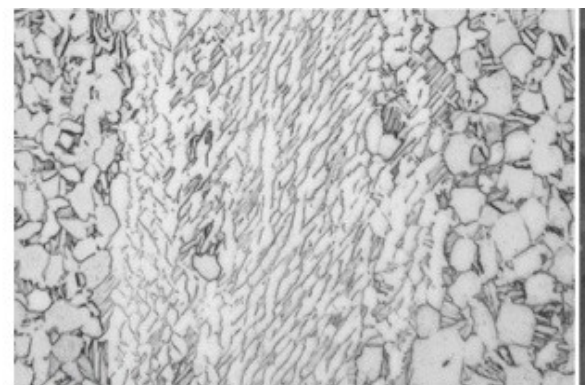

(a)

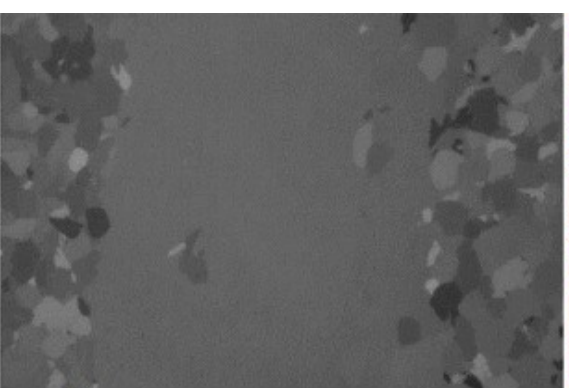

(b)

\section{$50 \mu \mathrm{m}$}

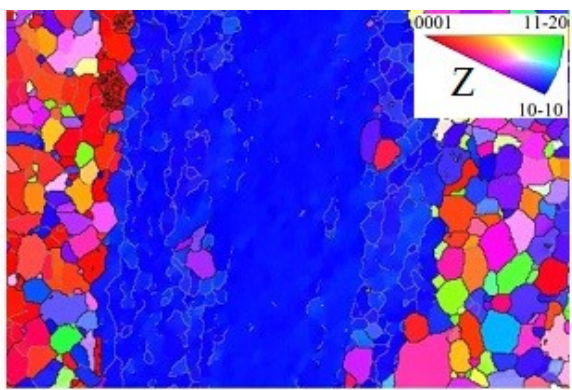

(c)

Figure 1. The microstructure of one microstructural variant observed viewed by bright field illumination in the etched condition (a) and polarized light in the un-etched condition (b) and EBSD (c) in the as polished condition.

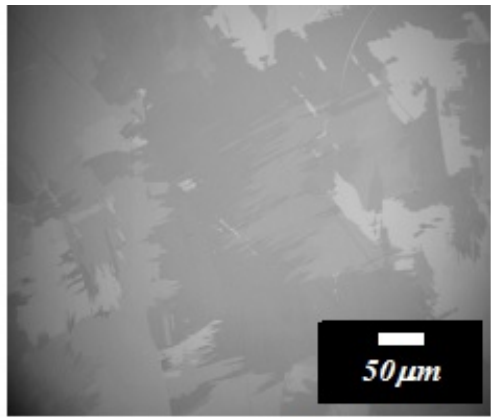

$0^{\circ}$

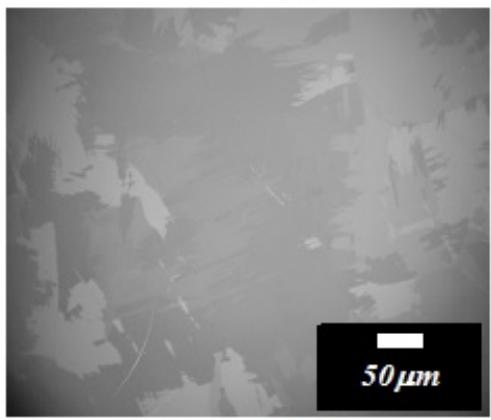

$180^{\circ}$

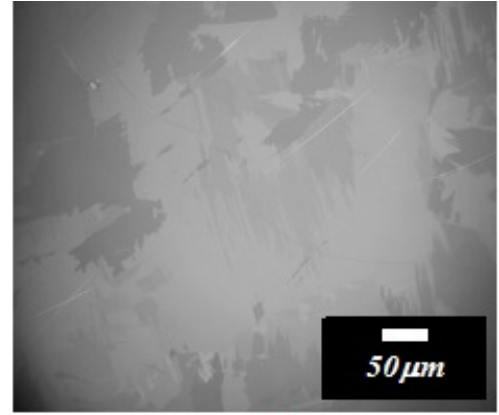

$90^{\circ}$

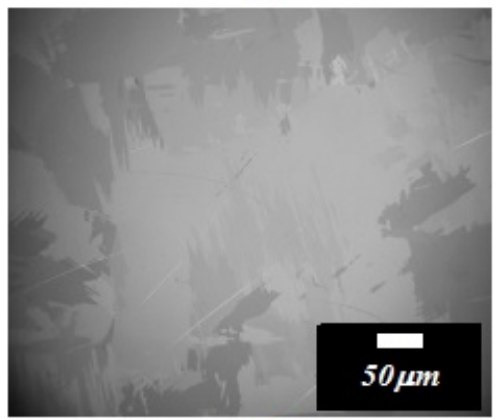

$270^{\circ}$

Figure 2. Qualitative observations of symmetry in the contrast of images recorded under polarised light condition.

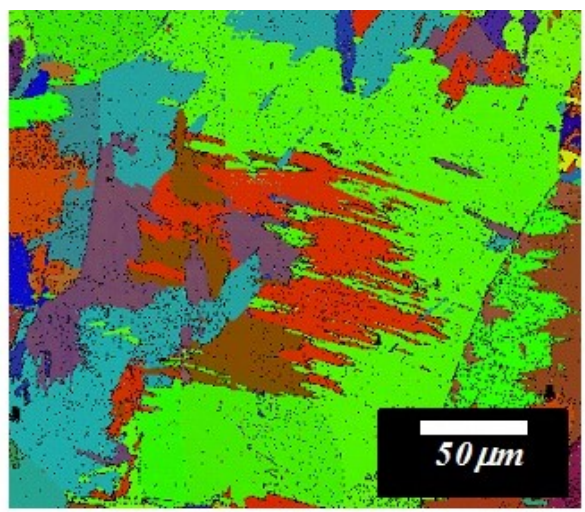

(a)

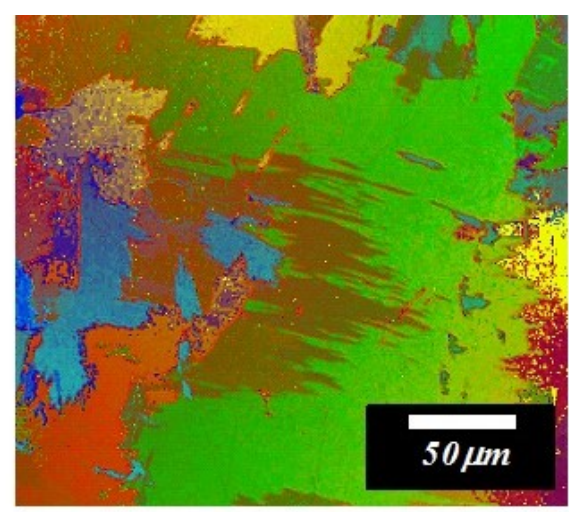

(b)

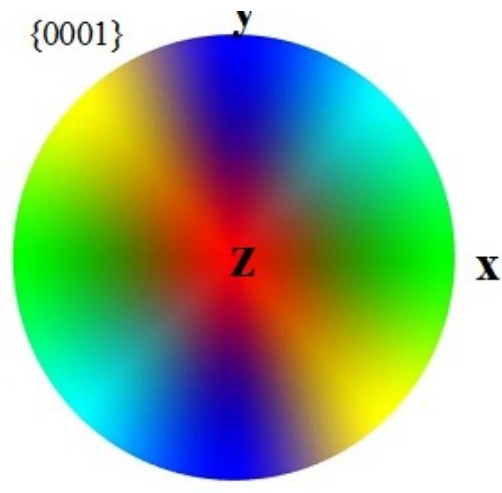

(c)

Figure 3. A comparison between basal pole figure maps produced via EBSD (a) and quantitative PLM (b). Maps are pole figure coloration (stereographic projection) denoted by key (c). 